\title{
Variable selection from a feature representing protein sequences: a case of classification on bacterial type IV secreted effectors
}

\author{
Jian Zhang ${ }^{1}$, Lixin LV1', Donglei Lu', Denan Kong ${ }^{2}$, Mohammed Abdoh Ali Al-Alashaari and Xudong Zhao ${ }^{2^{*}}$ (1)
}

\author{
*Correspondence: \\ zhaoxudong@nefu.edu.cn \\ ${ }^{2}$ College of Information \\ and Computer Engineering, \\ Northeast Forestry University, \\ No. 26 Hexing Road, \\ Harbin 150040, China \\ Full list of author information \\ is available at the end of the \\ article
}

\begin{abstract}
Background: Classification of certain proteins with specific functions is momentous for biological research. Encoding approaches of protein sequences for feature extraction play an important role in protein classification. Many computational methods (namely classifiers) are used for classification on protein sequences according to various encoding approaches. Commonly, protein sequences keep certain labels corresponding to different categories of biological functions (e.g., bacterial type IV secreted effectors or not), which makes protein prediction a fantasy. As to protein prediction, a kernel set of protein sequences keeping certain labels certified by biological experiments should be existent in advance. However, it has been hardly ever seen in prevailing researches. Therefore, unsupervised learning rather than supervised learning (e.g. classification) should be considered. As to protein classification, various classifiers may help to evaluate the effectiveness of different encoding approaches. Besides, variable selection from an encoded feature representing protein sequences is an important issue that also needs to be considered.
\end{abstract}

Results: Focusing on the latter problem, we propose a new method for variable selection from an encoded feature representing protein sequences. Taking a benchmark dataset containing 1947 protein sequences as a case, experiments are made to identify bacterial type IV secreted effectors (T4SE) from protein sequences, which are composed of 399 T4SE and 1548 non-T4SE. Comparable and quantified results are obtained only using certain components of the encoded feature, i.e., position-specific scoring matix, and that indicates the effectiveness of our method.

Conclusions: Certain variables other than an encoded feature they belong to do work for discrimination between different types of proteins. In addition, ensemble classifiers with an automatic assignment of different base classifiers do achieve a better classification result.

Keywords: Feature selection, Variable importance, Accumulated scoring, Classification, Bacterial type IV secreted effectors 


\section{Background}

Feature extraction from protein sequences plays an important role in protein classification [1-4] of many areas, such as identification of plant pentatricopeptide repeat coding protein [5], prediction of bacterial type IV secreted effectors [6-9], identification of heat shock protein [10], prediction of mitochondrial proteins [11], etc. In general, prevailing encoding approaches of protein sequences for feature extraction include pseudo-amino acid composition (PseAAC) [10-22], position-specific scoring matrix (PSSM) [7, 2332], position-specific iterated blast (PSI-BLAST) [33-37] etc.

However, several problems do still exist and are listed as follows. First of all, it needs to be decided which encoding approach is more effective. In fact, this problem can be solved according to the results of classification on each encoding approach using various computational methods (i.e., known as classifiers in the field of machine learning or pattern recognition). In other words, the encoding approach corresponding to the most accurate classification result should be considered. Prevailing classifiers including random forest or decision tree classifier (RF or DTC) [1,38], gradient boosting machine (GBM) [39, 40], k-nearest-neighbor ( $\mathrm{kNN}$ ) [41, 42], linear discriminant analysis (LDA) [43, 44], logistic regression (LR) [45], multi-layer perceptron (MLP) [46, 47], naive bayesian (NB) $[5,48]$, support vector machine (SVM) $[49,50]$ are credible.

Secondly, it needs to be discussed whether protein classification is predictive or not, which is a little confused. Actually, classification labels have commonly been assigned to protein sequences in advance. If these labels are definitive, i.e., having been certified by biological experiments in advance, there won't be any need to predict the category of a protein sequence again. Conversely, unsupervised learning (e.g. clustering) rather than supervised learning (e.g. classification) should be considered, since these labels are undetermined. And that corresponds to protein prediction. However, prevailing methods are always confusing protein classification an protein prediction.

Thirdly, the extracted feature using an encoding approach is considered to be entirely effective. In fact, there may be only parts of the extracted feature that are effective. However, this phenomenon has been subjectively neglected. As a result, it is an important issue how to select certain components or variables from a feature that really helps to recognize proteins with specific functions. In other words, variable selection from a feature representing protein sequences is a new problem probably not yet avoidable, which may be more helpful to classification of different protein sequences.

In this paper, we propose a new method for variable selection from an encoded feature. The selection of a feature from an encoding approach is excluded from our method. Besides, no prediction work is executed. Focusing on components or variables of an encoded feature, we implement our method at seven steps as shown in Fig. 1. First of all, samples are divided in balance, which constitute a training and testing group. Secondly, a base classifier is automatically assigned in every resampling of the training group. Thirdly, the score of each variable in an encoded feature is accumulated through $r$ rounds of resampling, training and scoring. Fourthly, a scatter plot and corresponding order of variables with their accumulated scores in a descending order are obtained. Fifthly, $r$ rounds of training are made on resampling samples to achieve ensemble classifiers in each dimension (i.e., from one to full dimension of the encoded feature) according to variables incrementally added in the descending order. Sixthly, variable selection 


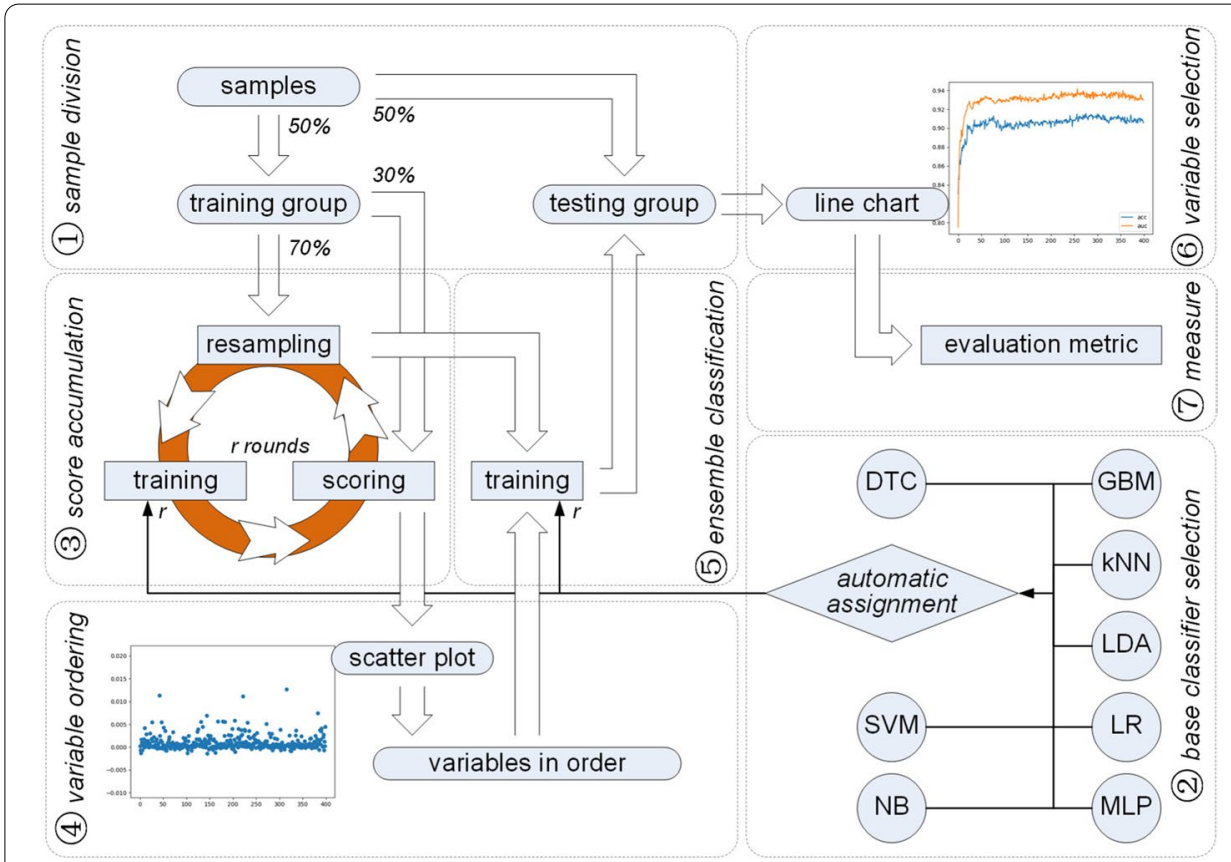

Fig. 1 A framework of variable selection for identifying different proteins

is accomplished using a line chart derived from the classification results on the testing group. Seventhly, evaluation metrics are made to estimate the effectiveness of selected variables. Experiments are made on the benchmark dataset [51] to identify bacterial type IV secreted effectors from protein sequences, which indicates the effectiveness of our method. More details can be seen in the following parts of this paper.

\section{Results}

In this section, we take a benchmark dataset [51] as a case to evaluate the performance of our proposed method. The dataset is composed of 1947 protein sequences across multiple bacterial species, categorized into two groups, i.e., 399 type IV secreted effectors (T4SE) as the positive samples and 1548 non-T4SE as the negative samples. The 1947 protein sequences are randomly divided into two subsets for training and testing, respectively. The training set consists of 973 sequences, among which 199 T4SE and 774 non-T4SE sequences are randomly selected from positive and negative samples, respectively. The left 200 T4SE and 774 non-T4SE samples constitute the testing set. Besides, we choose PSSM, which is composed of 400 variables, as the encoded feature. Following the procedure shown in Fig. 1, the experimental results of score accumulation, ensemble classification, variable selection and the corresponding classification results are listed as follows.

\section{Results of score accumulation}

We randomly extract $70 \%$ of samples from the training set and choose a classifier with the lowest classification error rate as the base classifier at each round. Meanwhile, scores representing the importance of variables are calculated. After 1000 rounds of resampling, training and scoring (i.e., $r=1000$ ), we obtain the accumulated scores of each 
variable from PSSM. The corresponding scatter plot is shown in Fig. 2a. Its horizontal and vertical coordinates correspond to 400 variables and their accumulated scores, respectively. In addition, the frequency of each selected base classifier is illustrated in Fig. 2b.

It can be seen in Fig. 2a that the accumulated scores are all relatively low. Since the accumulated scores of the 400 variables have no apparent distinction, all these variables are considered to be enumerated at the following step instead of selecting variables with high accumulated scores, as having been stated in [52].

In Fig. 2b, it can be seen that MLP is automatically assigned as the base classifier for $74.7 \%$ of 1000 round resampling. On the contrary, DTC and SVM have never been selected for score accumulation.

\section{Results of ensemble classification on testing group}

The ensemble classifiers have been built using 1000 rounds of resampling and training on the training set in each dimension, with 400 variables incrementally added in the descending order according to their accumulated scores. Then, the 400 ensemble classifiers, each of which keeps 1000 base classifiers, are applied to the testing set. As a result, a line chart (see Fig. 3) is obtained with its horizontal corresponding to the dimensions with variables incrementally added in the descending order according to their accumulated scores. The vertical coordinates are referred to the Acc and AUC values in

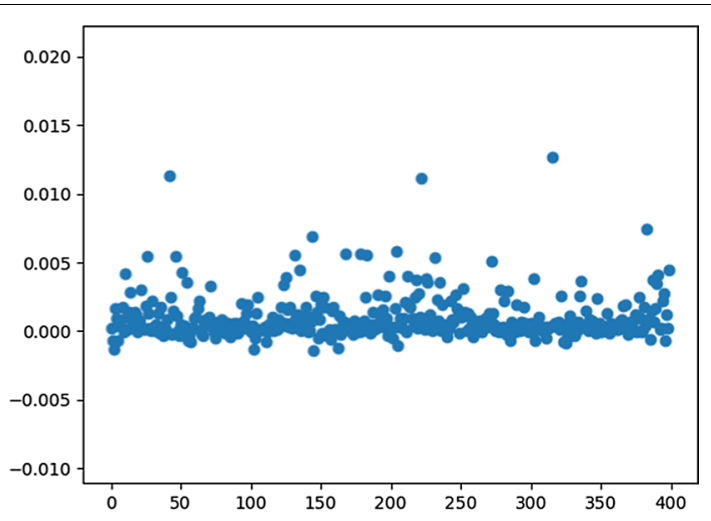

a A scatter plot of the accumulated scores corresponding to the variables from feature PSSM.

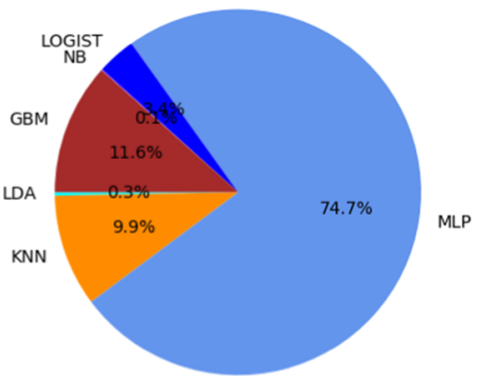

b A pan chart representing the frequency of each selected base classifier.

Fig. 2 Results of score accumulation 


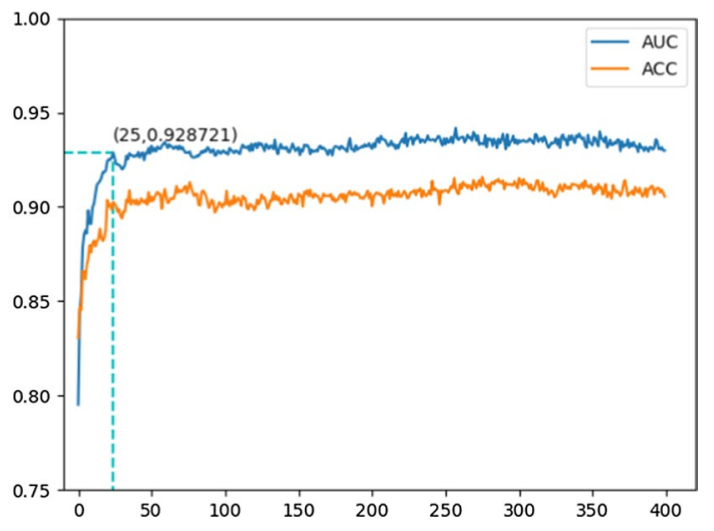

Fig. 3 A line chart of ACCs and AUCs corresponding to the incrementally added variables from feature PSSM with their accumulated scores in a descending order

the incremental dimensions. It can be seen in Fig. 3 that it is the first 25 variables with descending accumulated scores that form a 25-D feature from PSSM for effective identification of T4SE.

Moreover, some quantitative results are shown to indicate the effectiveness of the obtained 25 variables for classification. Table 1 lists the max, mean and min values of Accs and AUCs by incrementally adding the variables after the 25th one of the 400 variables in a descending order according to their accumulated scores. It can be indicated that features enlarged with higher dimensions can achieve only similar Accs and AUCs as the 25 variables do.

\section{Classification results of the selected variables}

In order to show the effectiveness of the selected 25 variables, the confusion matrix, Precision, Recall and $F 1$ - measure are calculated in order to make a quantitative comparison. In addition, ROCs together with AUCs are listed as qualitative results.

Results of ROC and AUC between the first 25 selected variables and all 400 components of PSSM using ensemble classification are shown in Fig. 4. The similar ROC curves and AUC values indicate that the selected 25 variables keep a comparable classification capability with PSSM.

Besides, results of ROC and AUC using the ensemble classifier consisting of 1000 single base classifiers with the selected 25 variables are illustrated in Fig. 5. By making a careful comparison between Figs. 4b and 5, it can be seen that ensemble classification with automatic assignment of base classifier keeps a better ROC curve and AUC value (i.e., 0.9287).

Moreover, quantitative results among ensemble classification with automatic assignment of a base classifier and the ensemble classifier with a single base classifier are listed in Table 2. It can be seen that the ensemble classifier with automatic assignment of a base classifier on the 25 selected variables keeps a high TP (i.e., 157) compared with most of the other classification strategies. Besides, it has better values of Precision, Recall and $F 1$ - measure (i.e., 0.904, 0.903 and 0.903) compared with the other ensemble classifier with a single base classifier on the 25 selected variables. As to the results of the ensemble 


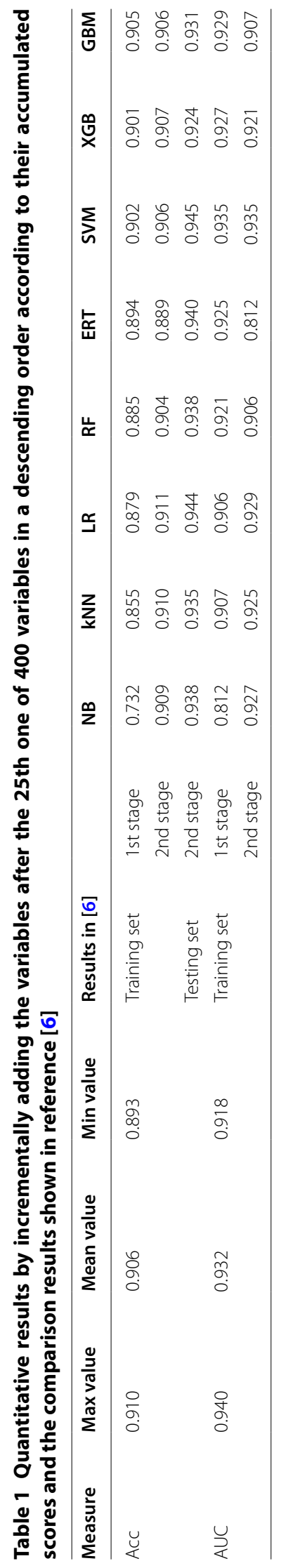


Table 2 Quantitative results among ensemble classification with automatic assignment of base classifiers and the ensemble classifier with single base classifiers

\begin{tabular}{|c|c|c|c|c|c|c|c|c|}
\hline \multirow{3}{*}{$\begin{array}{l}\text { Classifier } \\
\begin{array}{l}\text { Automatic } \\
\text { assignment }\end{array}\end{array}$} & \multirow{2}{*}{$\begin{array}{l}\text { Dimension } \\
400\end{array}$} & \multicolumn{3}{|c|}{ Confusion matrix } & \multirow{2}{*}{$\begin{array}{l}\begin{array}{l}\text { Positive } \\
\text { class }\end{array} \\
\text { Non-T4SE }\end{array}$} & \multirow{2}{*}{$\begin{array}{l}\text { Precision } \\
0.943\end{array}$} & \multirow{2}{*}{$\begin{array}{l}\text { Recall } \\
0.938\end{array}$} & \multirow{2}{*}{$\begin{array}{l}\text { F1-measure } \\
0.940\end{array}$} \\
\hline & & Classified as & Non-T4SE & T4SE & & & & \\
\hline & & $\begin{array}{l}\text { Label non- } \\
\text { T4SE }\end{array}$ & 726 & 48 & T4SE & 0.765 & 0.780 & 0.772 \\
\hline & & Label T4SE & 44 & 156 & $\begin{array}{r}\text { Weighted } \\
\text { average }\end{array}$ & 0.906 & 0.906 & 0.906 \\
\hline \multirow{3}{*}{$\begin{array}{l}\text { Automatic } \\
\text { assignment }\end{array}$} & 25 & Classified as & Non-T4SE & T4SE & Non-T4SE & 0.944 & 0.933 & 0.938 \\
\hline & & $\begin{array}{l}\text { Label non- } \\
\text { T4SE }\end{array}$ & 722 & 52 & T4SE & 0.751 & 0.785 & 0.768 \\
\hline & & Label T4SE & 43 & 157 & $\begin{array}{r}\text { Weighted } \\
\text { average }\end{array}$ & 0.904 & 0.903 & 0.903 \\
\hline \multirow[t]{3}{*}{ DTC } & 25 & Classified as & Non-T4SE & T4SE & Non-T4SE & 0.925 & 0.950 & 0.937 \\
\hline & & $\begin{array}{l}\text { Label non- } \\
\text { T4SE }\end{array}$ & 735 & 39 & T4SE & 0.782 & 0.700 & 0.739 \\
\hline & & Label T4SE & 60 & 140 & $\begin{array}{r}\text { Weighted } \\
\text { average }\end{array}$ & 0.896 & 0.899 & 0.896 \\
\hline \multirow[t]{3}{*}{ GBM } & 25 & Classified as & Non-T4SE & T4SE & Non-T4SE & 0.926 & 0.953 & 0.939 \\
\hline & & $\begin{array}{l}\text { Label non- } \\
\text { T4SE }\end{array}$ & 738 & 36 & T4SE & 0.797 & 0.705 & 0.748 \\
\hline & & Label T4SE & 59 & 141 & $\begin{array}{r}\text { Weighted } \\
\text { average }\end{array}$ & 0.900 & 0.902 & 0.900 \\
\hline \multirow[t]{3}{*}{ kNN } & 25 & Classified as & Non-T4SE & T4SE & Non-T4SE & 0.931 & 0.919 & 0.925 \\
\hline & & $\begin{array}{l}\text { Label non- } \\
\text { T4SE }\end{array}$ & 711 & 63 & T4SE & 0.700 & 0.735 & 0.717 \\
\hline & & Label T4SE & 53 & 147 & $\begin{array}{r}\text { Weighted } \\
\text { average }\end{array}$ & 0.884 & 0.881 & 0.882 \\
\hline \multirow[t]{3}{*}{ LDA } & 25 & Classified as & Non-T4SE & T4SE & Non-T4SE & 0.927 & 0.925 & 0.926 \\
\hline & & $\begin{array}{l}\text { Label non- } \\
\text { T4SE }\end{array}$ & 716 & 58 & T4SE & 0.713 & 0.720 & 0.716 \\
\hline & & Label T4SE & 56 & 144 & $\begin{array}{r}\text { Weighted } \\
\text { average }\end{array}$ & 0.883 & 0.883 & 0.883 \\
\hline \multirow[t]{3}{*}{ LR } & 25 & Classified as & Non-T4SE & T4SE & Non-T4SE & 0.883 & 0.957 & 0.919 \\
\hline & & $\begin{array}{l}\text { Label non- } \\
\text { T4SE }\end{array}$ & 741 & 33 & T4SE & 0.756 & 0.510 & 0.609 \\
\hline & & Label T4SE & 98 & 102 & $\begin{array}{r}\text { Weighted } \\
\text { average }\end{array}$ & 0.857 & 0.865 & 0.855 \\
\hline \multirow[t]{3}{*}{ MLP } & 25 & Classified as & Non-T4SE & T4SE & Non-T4SE & 0.930 & 0.946 & 0.938 \\
\hline & & $\begin{array}{l}\text { Label non- } \\
\text { T4SE }\end{array}$ & 732 & 42 & T4SE & 0.775 & 0.725 & 0.749 \\
\hline & & Label T4SE & 55 & 145 & $\begin{array}{r}\text { Weighted } \\
\text { average }\end{array}$ & 0.898 & 0.901 & 0.899 \\
\hline \multirow[t]{3}{*}{ NB } & 25 & Classified as & Non-T4SE & T4SE & Non-T4SE & 0.942 & 0.875 & 0.907 \\
\hline & & $\begin{array}{l}\text { Label non- } \\
\text { T4SE }\end{array}$ & 677 & 97 & T4SE & 0.620 & 0.790 & 0.695 \\
\hline & & Label T4SE & 42 & 158 & $\begin{array}{r}\text { Weighted } \\
\text { average }\end{array}$ & 0.876 & 0.858 & 0.863 \\
\hline \multirow[t]{3}{*}{ SVM } & 25 & Classified as & Non-T4SE & T4SE & Non-T4SE & 0.925 & 0.924 & 0.924 \\
\hline & & $\begin{array}{l}\text { Label non- } \\
\text { T4SE }\end{array}$ & 715 & 59 & T4SE & 0.706 & 0.710 & 0.708 \\
\hline & & Label T4SE & 58 & 142 & $\begin{array}{r}\text { Weighted } \\
\text { average }\end{array}$ & 0.880 & 0.880 & 0.880 \\
\hline
\end{tabular}




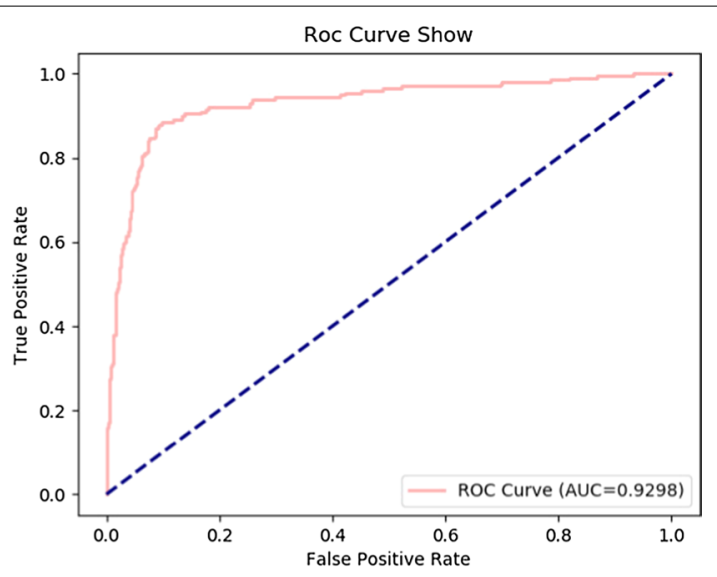

a Results of all 400 components.

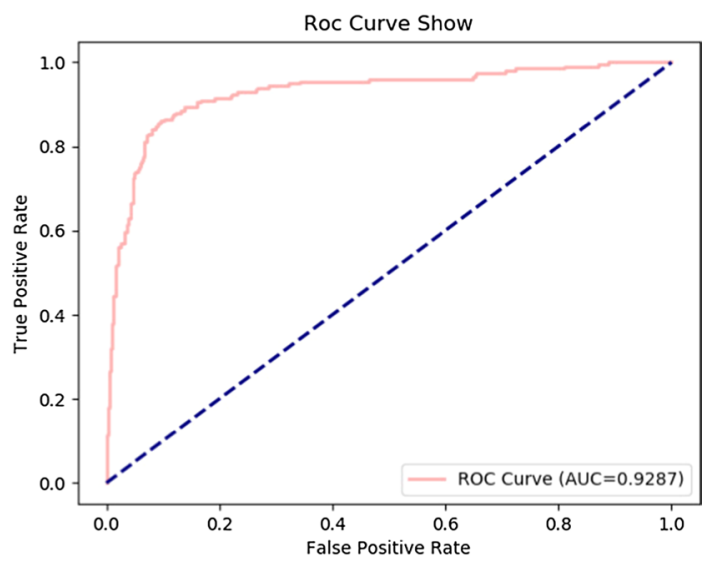

b Results of the selected 25 variables.

Fig. 4 Comparison results of ROC and AUC between all 400 components and the selected 25 variables using ensemble classification

classifier with automatic assignment of a base classifier on PSSM (i.e., 400 components), the selected 25 variables can also achieve comparable results.

\section{Discussions}

Experimental results have indicated the effectiveness of variable selection from the encoded feature PSSM. In this section, we will further discuss the special composition of our variable selection method and the classification results.

\section{Purpose of using base classifier selection}

The automatic assignment of a base classifier is creative in this paper. Giving consideration to the sample distribution of resampling, we designed the strategy of automatic assignment. Due to the limited sample size, resampling is only an approximation to the population. In our previous work, it has been pointed out that different base classifiers should be considered according to various sample distributions [52]. However, the base classifier was interactively appointed in [52]. The pan chart in Fig. 2b can also show this phenomenon, which indicates that base classifiers selected at a higher percentage 


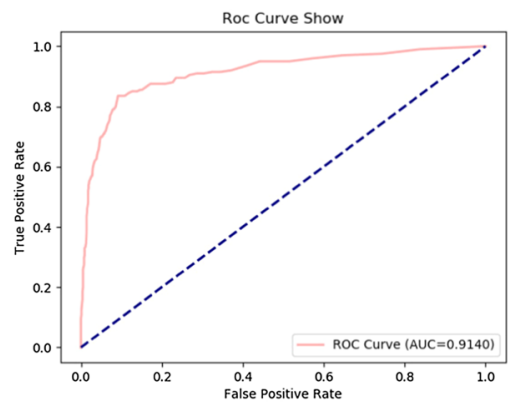

a Results using DTC.

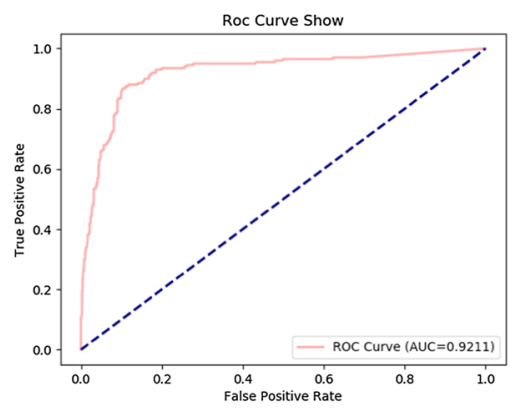

c Results using $\mathrm{kNN}$.

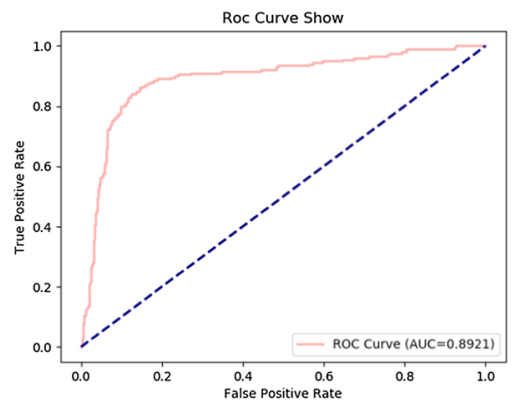

e Results using LR.

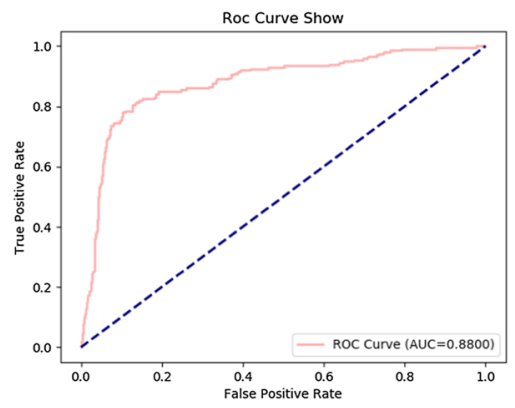

g Results using NB.

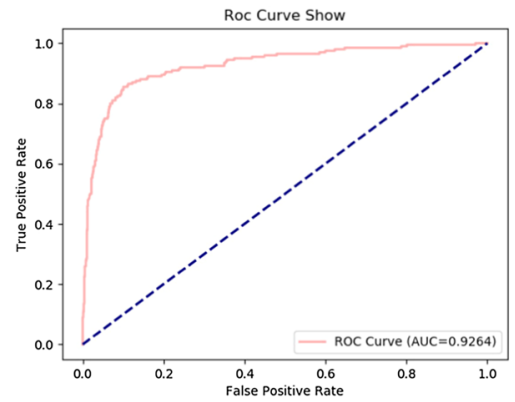

b Results using GBM.

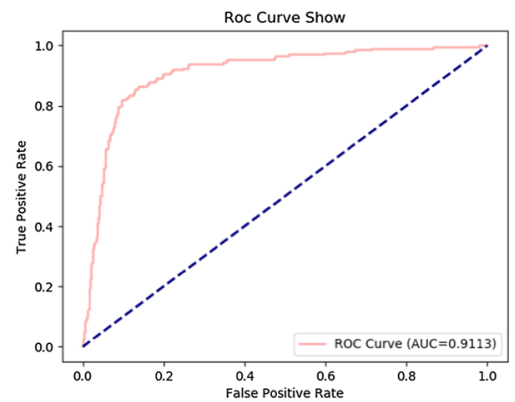

d Results using LDA.

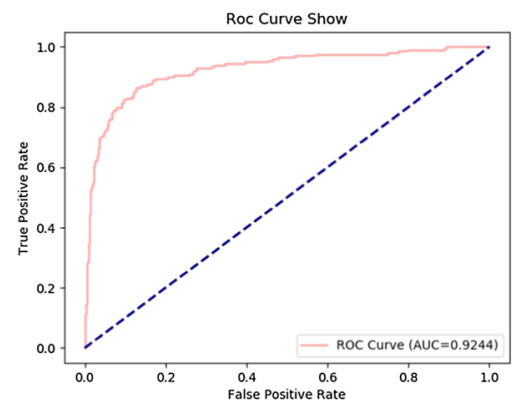

f Results using MLP.

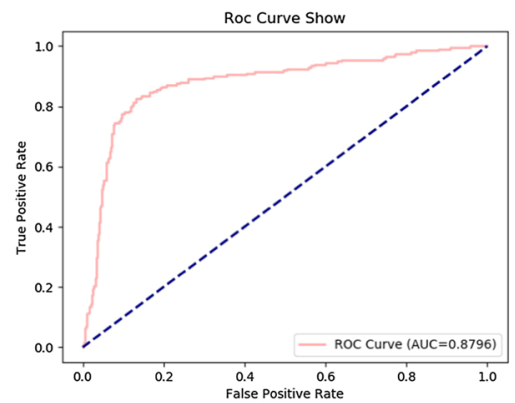

h Results using SVM.

Fig. 5 Results of ROC and AUC using different base classifiers with the selected 25 variables 
may fit the population better. Besides, quantitative results listed in Table 2 indicate the power of using automatic assignment compared with the interactive appointment of a base classifier.

\section{Purpose of using a line chart for variable selection}

In fact, a line chart shown in Fig. 3 is a semi-automatic way for variable selection. Here, it goes against the interactive way that uses a manual selection within a table or on a 2-D scatter plot [52]. Also, it abandons the automatic way of automatic clustering [53] on accumulated scores. Due to the limited distribution of the accumulated scores with relatively low values, the variables have no apparent distinction. It probably means variables are highly correlated. In that case, variable orders instead of values are to be considered.

\section{Comparisons between classification results}

We compared the classification results of our method with PredT4SE-Stack [6]. As shown in Table 1, the max, mean and min values of Accs and AUCs on the testing set exhibit a better result than the classification results on its training set using PreT4SEStack. The best classification Acc value using PredT4SE-Stack on its training set is 0.911 . Most of the other meta-classifier got a lower Acc value and AUC value than the mean Acc and AUC obtained using our method. However, classification results of Pred4SEStack on its testing set are better than those using our method. In fact, the classification results on its testing set are even better than those on its training set. By careful observation, it is found that parameters of base classifiers are manually set every time in [6]. That's why PredT4SE-Stack gets better classification results on its testing set. Anyway, seeking better classification results by setting parameter values doesn't make any sense for variable selection.

\section{Conclusion}

In order to solve the problem of variable selection from an encoded feature representing protein sequences, we propose a variable selection method based on ensemble classification with an automatic assignment of base classifiers. Variable ordering is obtained according to score accumulation on training samples. Then, ensemble classifiers are established from one to all dimensions of the encoded feature according to variables incrementally added in the descending order. Using the ensemble classifiers on testing samples, a line chart is drawn for variable selection. Ultimately, evaluation metrics are made to estimate the effectiveness of selected variables. Taking a dataset containing protein sequences categorized into T4SE and non-T4SE group as a case, the performance of the proposed method is evaluated.

\section{Methods}

In this section, we will expound our method in detail. As illustrated in Fig. 1, our method has seven steps, each of which is framed in a dashed box. Each step which keeps its name labeled within the dashed box, corresponds to a following subsection. 


\section{Sample division}

We make a balanced sample division at the first step. That is, samples derived from a category are divided into two equivalent parts. As a result, half of the samples from different categories form a training group; while, the left ones constitute a testing group. It is noteworthy that this sample division should be performed in a completely random way.

\section{Base classifier selection}

A base classifier is automatically assigned at the second step. We make a set of base classifiers including decision tree classifier (DTC), gradient boosting machine (GBM), k-nearest-neighbor $(\mathrm{kNN})$, linear discriminant analysis (LDA), logistic regression (LR), multi-layer perceptron (MLP), naive bayesian (NB) and support vector machine (SVM). Each one is equally assigned in an automatic way corresponding to every round of resampling and training module. In each round $j, 70 \%$ of training samples are randomly selected in a full dimension of an encoded feature for training each base classifier. The remaining $30 \%$ of training samples are regarded as the out-of-bag samples for calculation of the classification error rate Err , as is expressed in Eq. (1). The base classifier with the lowest classification error rate is automatically assigned in round $j$.

\section{Score accumulation}

Score accumulation is made at the third step. Once a base classifier is automatically assigned according to the classification error rate calculated on the out-of-bag samples in round $j$, permutations are to be made. As to each variable $i$ of the encoded feature, only one-time permutation of the expression values from the out-of-bag samples is performed. The corresponding classification error rate is denoted as $\operatorname{Err}_{j}^{0}(i)$. Accordingly, a score representing the importance of variable $i$ is expressed as $\operatorname{score}_{j}(i)=\operatorname{Err}_{j}^{0}(i)-\operatorname{Err}_{j}$. After $r$ rounds of resampling, training and scoring, the accumulated score of variable $i$ is expressed as $\sum_{j=1}^{r} \operatorname{score}_{j}(i) / r$.

\section{Variable ordering}

Variables are reordered at the fourth step. A 2-D scatter plot is to be made with its horizontal and vertical coordinates corresponding to the variable indices and the accumulated scores, respectively. Besides, variables are to be sorted in a descending order according to the accumulated scores. If the accumulated scores of the variables have no distinction (i.e., the accumulated scores are all relatively low), all the variables rather than the significant variables selected using previously proposed clustering method [53] are to be enumerated at the following step.

\section{Ensemble classification}

Ensemble classifiers are established at the fifth step. Again, $r$ rounds of resampling and training are performed to achieve ensemble classifiers in each dimension according to variables incrementally added in their descending order. As to 1-D space, the variable with the highest accumulated score is considered. At each round of resampling, the base classifier with the lowest classification error rate is trained. Altogether, $r$ base 
classifiers are selected as the ensemble classifier in 1-D space. This procedure is repeated with a variable keeping a lower accumulated score added, until the full dimension of the encoded feature or the full dimension of significant variables has been considered.

\section{Variable selection}

Variable selection is accomplished at the sixth step. In each dimension, the established ensemble classifier is applied to the testing samples. The accuracy (Acc) expressed in Eq. (2) and the area under curve (AUC) of the receiver operating characteristic (ROC) are calculated. Accordingly, a line chart is obtained with its horizontal and vertical coordinates corresponding to the variable indices in their descending order and the corresponding Accs and AUCs in different dimensions. A dimension threshold can be made when Accs and AUCs are keeping almost the same with dimension incrementally increasing. Thus, the variables that really help to recognize proteins with specific functions are selected from the encoded feature.

\section{Measure}

Evaluation metrics are made to estimate the effectiveness of selected variables at the seventh step. The classification error rate is expressed as follows,

$$
E r r=\frac{F N+F P}{T P+F N+T N+F P},
$$

where TP, TN, FP and $F N$ represent the number of true positive, true negative, false positive and false negative, respectively. On the contrary, Acc is shown as follows,

$$
A c c=\frac{T N+T P}{T P+F N+T N+F P} .
$$

Besides, we choose four widely used quantitative measurements. The confusion matrix illustrates TP, TN, FP and FN together. Besides, Precision and Recall are computed as follows,

$$
\begin{aligned}
& \text { Precision }=\frac{T P}{T P+F P}, \\
& \text { Recall }=\frac{T P}{T P+F N} .
\end{aligned}
$$

In addition, F1-measure is a harmonic average of Precision and Recall, which is expressed as

$$
F 1-\text { measure }=\frac{2 * \text { Precision } * \text { Recall }}{\text { Precision }+ \text { Recall }} .
$$

Moreover, the ROC and AUC are also provided here as qualitative measurements.

\section{Abbreviations}

Acc:: Accuracy; AUC:: Area under curve; DTC:: Decision tree classifier;: GBM:: Gradient boosting machine; kNN:: k-nearestneighbor;: LDA:: Linear discriminant analysis;; LR:: Logistic regression;; MLP:: Multi-layer perceptron;; NB:: Naive bayesian;" PseAAC:: Pseudo-amino acid composition;; PSI-BLAST:: Position-specific iterated blast; PSSM:: Position-specific scoring 
matrix:; RF:: Random forest;: ROC:: Receiver operating characteristic;: SVM::: Support vector machine;:; TSEE:: Type IV secreted effectors.

\section{Acknowledgements}

This work is derived from Scientific Research Project Supported by Enterprise Suzhou Dachen Medical Technology Co. Ltd.

\section{Authors' contributions}

X.D.Z conceived the general research and supervised it. J.Z performed the research and were the principal developers. D.L.L and L.X.L analyzed the data. D.N.K, M.A.A.A and X.D.Z wrote and revised the manuscript. All authors read and approved the final manuscript.

\section{Funding}

This work has been supported by the financial support of This work has been supported by the financial support of Natural Science Foundation of Heilongjiang Province (No. LH2020F002). The funding body of Fundamental Research Funds for Natural Science Foundation of Heilongjiang Province played an important role in the design of the study, collection, analysis and interpretation of data and in writing the manuscript.

\section{Availability of data and materials}

The public dataset analysed during the current study is available in reference [51], and can be downloaded from the website https://github.com/LoopGan/Effective-prediction-of-bacterial-type-IV-secreted-effectors.

\section{Ethics approval and consent to participate}

Not applicable.

\section{Consent for publication}

Not applicable.

\section{Competing interests}

The authors declare that they have no known competing financial interests or personal relationships that could have appeared to influence the work reported in this paper.

\section{Author details}

${ }^{1}$ College of Artificial Intelligence, Wuxi Vocational College of Science and Technology, No. 8 Xinxi Road, Wuxi 214028 China. ${ }^{2}$ College of Information and Computer Engineering, Northeast Forestry University, No. 26 Hexing Road, Harbin 150040 , China.

Received: 21 July 2020 Accepted: 19 October 2020

Published online: 27 October 2020

\section{References}

1. Lv ZB, Jin SS, Ding H, Zou Q. A random forest sub-Golgi protein classifier optimized via dipeptide and amino acid composition features. Fronti Bioeng Biotechnol. 2019;7:215.

2. Zhu XJ, Feng CQ, Lai HY, Chen W, Lin H. Predicting protein structural classes for low-similarity sequences by evaluating different features. Knowl-Based Syst. 2019;163:787-93.

3. Ru XQ, Li LH, Zou Q. Incorporating distance-based top-n-gram and random forest to identify electron transport proteins. J Proteome Res. 2019;18:2931-9.

4. Li YJ, Niu MT, Zou Q. ELM-MHC: an improved MHC identification method with extreme learning machine algorithm. J Proteome Res. 2019;18:1392-401.

5. Qu K, Wei L, Yu J, Wang C. Identifying plant pentatricopeptide repeat coding gene/protein using mixed feature extraction methods. Front Plant Sci. 2019:9:1-10.

6. Xiong Y, Wang QK, Yang JC, Zhu XL, Wei DQ. PredT4SE-Stack: prediction of bacterial type IV secreted effectors from protein sequences using a stacked ensemble method. Front Microbiol. 2018;9:2571.

7. Zou LY, Nan CH, Hu FQ. Accurate prediction of bacterial type IV secreted effectors using amino acid composition and PSSM profiles. Bioinformatics. 2013;29(24):3135-42.

8. Ashari ZE, Dasgupta N, Brayton KA, Broschat SL. An optimal set of features for predicting type IV secretion system effector proteins for a subset of species based on a multi-level feature selection approach. PLOS ONE. 2018;13:e0197041.

9. Yu LZ, Guo YZ, Li YZ, Li GB, Li ML, Luo JS, Xiong WJ, Qin WL. SecretP: identifying bacterial secreted proteins by fusing new features into Chou's pseudo-amino acid composition. J Theor Biol. 2010;267:1-6.

10. Feng PM, Chen W, Lin H, Chou KC. iHSP-PseRAAAC: identifying the heat shock protein families using pseudo reduced amino acid alphabet composition. Anal Biochem. 2013;442(1):118-25.

11. Mirza MT, Khan A, Tahir M, Lee YS. MitProt-Pred: predicting mitochondrial proteins of Plasmodium falciparum parasite using diverse physiochemical properties and ensemble classification. Comput Biol Med. 2013;43(10):1502-11.

12. Ahmad J, Hayat M. MFSC: multi-voting based feature selection for classification of Golgi proteins by adopting the general form of Chou's PseAAC components. J Theor Biol. 2019;463:99-109.

13. Zhang SL, Duan X. Prediction of protein subcellular localization with oversampling approach and Chou's general PseAAC. J Theor Biol. 2018:437:239-50. 
14. Srivastava A, Kumar R, Kumar M. BlaPred: predicting and classifying beta-lactamase using a 3-tier prediction system via Chou's general PseAAC. J Theor Biol. 2018;457:29-36.

15. Sankari ES, Manimegalai D. Predicting membrane protein types by incorporating a novel feature set into Chou's general PseAAC. J Theor Biol. 2018;455:319-28.

16. Sankari ES, Manimegalai D. Predicting membrane protein types using various decision tree classifiers based on various modes of general PseAAC for imbalanced datasets. J Theor Biol. 2017:435:208-17.

17. Liang YY, Zhang SL. Predict protein structural class by incorporating two different modes of evolutionary information into Chou's general pseudo amino acid composition. J Mol Graph Model. 2017;78:1 10-7.

18. Meher PK, Sahu TK, Banchariya A, Rao AR. DIRProt: a computational approach for discriminating insecticide resistant proteins from non-resistant proteins. BMC Bioinform. 2017;18:190.

19. Tiwari AK. Prediction of $G$-protein coupled receptors and their subfamilies by incorporating various sequence features into Chou's general PseAAC. Comput Methods Programs Biomed. 2016;134:197-213.

20. Han GS, Yu ZG, Anh V. A two-stage SVM method to predict membrane protein types by incorporating amino acid classifications and physicochemical properties into a general form of Chou's PseAAC. J Theor Biol. 2014;344:31-9.

21. Chou K. Using amphiphilic pseudo amino acid composition to predict enzyme subfamily classes. Bioinformatics. 2005;21:10-9.

22. Chou K. Prediction of protein cellular attrbutes using pseudo-amino acid composition. Proteins. 2001;43:246-55

23. Wang JW, Yang BJ, Revote J, Leier A, Marquez-Lago TT, Webb G, Song JN, Chou KC, Lithgow T. POSSUM: a bioinformatics toolkit for generating numerical sequence feature descriptors based on PSSM profiles. Bioinformatics. 2017;33(17):2756-8.

24. Zhang LC, Zhao XQ, Kong L. Predict protein structural class for low-similarity sequences by evolutionary difference information into the general form of Chou's pseudo amino acid composition. J Theor Biol. 2014;355:105-10

25. Paliwal KK, Sharma A, Lyons J, Dehzangi A. A tri-gram based feature extraction technique using linear probabilities of position specific scoring matrix for protein fold recognition. IEEE Trans Nanobiosci. 2014;13(1):44-50.

26. Zahiri J, Yaghoubi O, Mohammad-Noori M, Ebrahimpour R, Masoudi-Nejad A. PPlevo: protein-protein interaction prediction from PSSM based evolutionary information. Genomics. 2013;102(4):237-42.

27. Zhang SL, Ye F, Yuan XG. Using principal component analysis and support vector machine to predict protein structural class for low-similarity sequences via PSSM. J Biomol Struct Dyn. 2012;29(6):634-42.

28. Jeong JC, Lin XT, Chen XW. On position-specific scoring matrix for protein function prediction. IEEE-ACM Trans Comput Biol Bioinform. 2011;8(2):308-15.

29. Jia CZ, Liu T, Chang AK, Zhai YY. Prediction of mitochondrial proteins of malaria parasite using bi-profile Bayes feature extraction. Biochimie. 2011;93(4):778-82.

30. Dong QW, Zhou SG, Guan JH. A new taxonomy-based protein fold recognition approach based on autocrosscovariance transformation. Bioinformatics. 2009;25(20):2655-62.

31. Cheng CW, Su ECY, Hwang JK, Sung TY, Hsu WL. Predicting RNA-binding sites of proteins using support vector machines and evolutionary information. BMC Bioinform. 2008;9(S12):S6.

32. Chou KC, Shen HB. MemType-2L: a web server for predicting membrane proteins and their types by incorporating evolution information through Pse-PSSM. Biochem Biophys Res Commun. 2007;360(2):339-45.

33. An JY, You ZH, Chen X, Huang DS, Li ZW, Liu G, Wang Y. Identification of self-interacting proteins by exploring evolutionary information embedded in PSI-BLAST-constructed position specific scoring matrix. Oncotarget. 2016;7(50):82440-9.

34. Qin YF, Zheng XQ, Wang J, Chen M, Zhou CJ. Prediction of protein structural class based on Linear Predictive Coding of PSI-BLAST profiles. Open Life Sciences. 2015;10(1):529-36.

35. Ding SY, Li Y, Shi ZX, Yan SJ. A protein structural classes prediction method based on predicted secondary structure and PSI-BLAST profile. Biochimie. 2014;97:60-5.

36. Liu T, Zheng XQ, Wang J. Prediction of protein structural class for low-similarity sequences using support vector machine and PSI-BLAST profile. Biochimie. 2010;92(10):1330-4.

37. Kaur H, Raghava GPS. Prediction of alpha-turns in proteins using PSI-BLAST profiles and secondary structure information. Proteins-Struct Funct Bioinform. 2004;55(1):83-90.

38. Breiman L. Random forests. Mach Learn. 2001;45:5-32.

39. Tan CG, Wang T, Yang WY, Deng L. PredPSD: a gradient tree boosting approach for single-stranded and doublestranded DNA binding protein prediction. Molecules. 2020;25(1):98.

40. Friedman JH. Greedy function approximation: a gradient boosting machine. Ann Stat. 2001:29(5):1189-232.

41. He ZY, Liu H, Moch H, Simon H. Machine learning with autophagy-related proteins for discriminating renal cell carcinoma subtypes. Sci Rep. 2020;10(1):720.

42. Cover T, Hart P. Nearest neighbor pattern classification. IEEE Trans Inf Theory. 1967;13(1):21-7.

43. Isopescu RD, Spulber R, Josceanu AM, Mihaiescu DE, Popa O. Romanian bee pollen classification and property modelling. J Apicult Res. 2020.

44. Belhumeur PN, Hespanha JP, Kriegman DJ. Eigenfaces vs. fisherfaces: recognition using class specific linear projection. IEEE Trans Pattern Anal Mach Intell. 1997;19(7):711-20.

45. Wachters JE, Kop E, Slagter-Menkema L, Mastik M, van der Wal JE, van der Vegt B. de Bock GH, van der Laan BFAM, Schuuring E. Distinct biomarker profiles and clinical characteristics in T1-T2 glottic and supraglottic carcinomas. The Laryngoscope 2020.

46. Zhou Y, Li GQ, Li HQ. Automatic cataract classification using deep neural network with discrete state transition. IEEE Trans Med Imaging. 2020;39(2):436-46.

47. Pal SK, Mitra S. Multilayer perceptron, fuzzy sets, and classification. IEEE Trans Neural Netw. 1992;3(5):683-97.

48. Domingos P, Pazzani M. On the optimality of the simple Bayesian classifier under zero-one loss. Mach Learn. 1997:29(2-3):103-30. 
49. Meng CL, Jin SS, Wang L, Guo F, Zou Q. AOPs-SVM: a sequence-based classifier of antioxidant proteins using a support vector machine. Front Bioeng Biotechnol 2019.

50. Cortes C, Vapnik VN. Support vector networks. Mach Learn. 1995;20(3):273-97.

51. Wang $Y$, GuO Y, Pu X, Li M. Effective prediction of bacterial type IV secreted effectors by combined features of both C-termini and N-termini. J Comput Aided Mol Des. 2017;31:1029-38.

52. Zhao XD, Jiao Q, Li HY, Wu YM, Wang HX, Huang S, Wang GH. ECFS-DEA: an ensemble classifier-based feature selection for differential expression analysis on expression profiles. BMC Bioinform. 2020;21:43.

53. LiU T, Li HY, Zhao XD. Clustering by search in descending order and automatic find of density peaks. IEEE Access. 2019;7:133772-80.

\section{Publisher's Note}

Springer Nature remains neutral with regard to jurisdictional claims in published maps and institutional affiliations.

- fast, convenient online submission

- thorough peer review by experienced researchers in your field

- rapid publication on acceptance

- support for research data, including large and complex data types

- gold Open Access which fosters wider collaboration and increased citations

- maximum visibility for your research: over 100M website views per year

At BMC, research is always in progress.

Learn more biomedcentral.com/submissions 